\title{
The role of galaxy merging in the life of massive galaxies
}

\author{
Allison W. S. Man \\ European Southern Observatory (Garching) \\ email: allison.man@eso.org
}

\begin{abstract}
The role of galaxy mergers in the evolution of massive galaxies remains debated. While deep near-infrared surveys have enabled several independent merger rate measurements out to $z \sim 3$, they are limited to small samples and results are discrepant at $z=2-3$. In Man et al., we use the UltraVISTA and CANDELS surveys to obtain the largest sample of photometric galaxy pairs at $z>1$ for measuring the galaxy merger fraction and rate of massive galaxies. We find that the discrepancy of previous studies is due to selection effect. Defining galaxy pairs by stellar mass ratio leads to a flat $z$-evolution of the merger fraction, while defining by flux ratio leads to an increasing trend. The implications on the evolution of massive galaxies are summarized here.
\end{abstract}

Keywords. galaxies: evolution, galaxies: high-redshift, galaxies: interactions, galaxies: statistics

Galaxy major mergers in the local Universe trigger the most luminous starbursts and active galactic nuclei (e.g., Kartaltepe et al. 2010; Treister et al. 2012). Additionally, compact massive galaxies assembled and quenched at $z \gtrsim 2$ are expected grow stellar envelops to become present-day massive ellipticals, through a series of gas-poor minor mergers (Naab et al. 2009). In order to assess the importance of galaxy merging in the evolution of massive galaxies, the galaxy merger rate needs to be constrained. Galaxy pairs are used to probe pre-coalescence major and minor merger candidates out to $z \sim 3$. Major (minor) mergers are usually defined as having mass ratios between 1:1 and 1:4 (1:4 and 1:10). The galaxy merger fraction, as a function of $z$, is then divided by merger observability timescale (Lotz et al. 2010) to obtain the galaxy merger rate.

The method described above requires deep and wide surveys covering optical and nearinfrared (NIR) wavelengths. Tension exists among the few measurements of the galaxy merger rate evolution beyond $z \gtrsim 2$, for the most massive $\left(M_{\star} \gtrsim 10^{10.8} M_{\odot}\right)$ galaxies: the galaxy major merger fractions are found to increase with redshift in some works (Bluck et al. 2009; Man et al. 2012), while it remains flat or even diminishing towards high- $z$ in others (Williams et al. 2011; Newman et al. 2012). However, it was unclear whether the discrepancy originates from the difference in datasets (ground-based vs space-based), merger mass ratio definition (stellar mass ratio vs observed $H$-band flux ratio), or small number statistics.

In Man et al. (2014), we have resolved the discrepancy in previous galaxy merger fraction measurements. Using the ground-based NIR survey UltraVISTA (McCracken et al. 2012; Ilbert et al. 2013), complemented with the HST CANDELS survey (Grogin et al. 2011; Koekemoer et al. 2011), we perform an extensive analysis on the largest sample of photometrically selected galaxy pairs $(>1000)$. The large sample size reduces the random uncertainties, which were the dominant source of errors in previous measurements of the galaxy merger rate. In short, the discrepant measurements of the galaxy merger fraction at $z \sim 2$ is due to the merger mass ratio probe (Fig. ). Defining galaxy mergers by the observed $H$-band flux ratio leads to a bias towards bright star-forming satellites at $z \sim 2$. 


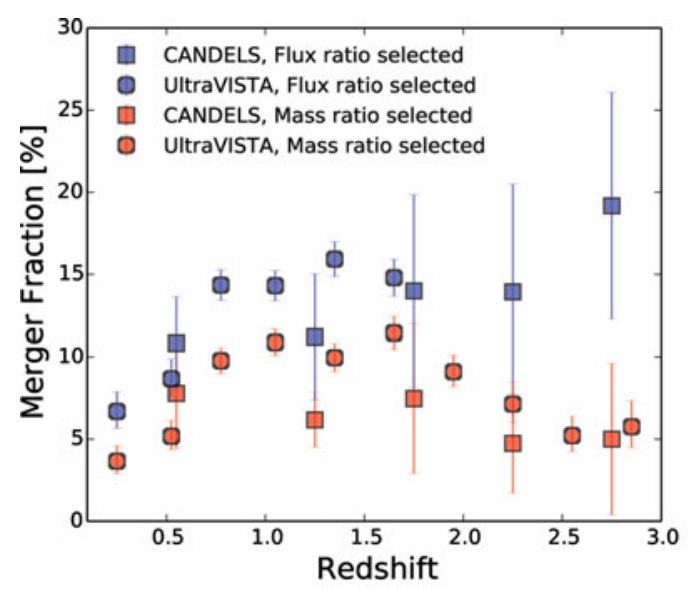

(a)

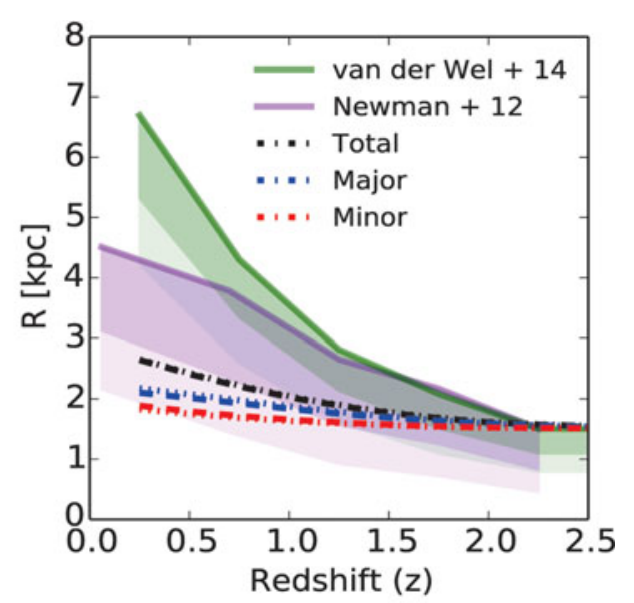

(b)

Figure 1. (a) Using the same datasets, the major merger fraction increases with redshift if mergers are selected using the $H$-band flux ratio (blue), and diminishes if the stellar mass ratio is used instead (red). (b) The predicted size evolution (black line) of massive quiescent galaxies driven by the observed galaxy merger rate (major + minor), compared to the observed size evolution (plotted as median lines, and the shades indicate $1 \times$ and $2 \times$ dispersions) presented in literature.

In contrast, using the stellar mass ratio to define mergers is biased against such satellites with considerable cold gas mass.

Converting the galaxy merger fraction into merger rate, we find that the observed merger rates are lower than the predicted ones from semi-analytical models, with a larger discrepancy towards higher $z$. As the gas fraction of galaxies varies with $M_{\star}$ and $z, M_{\star}$ is increasingly inadequate for tracing baryon / dynamical / halo mass at high $z$ and low $M_{\star}$. In another words, using stellar mass ratio to define mergers drawn from $M_{\star}$-limited samples leads to an underestimation of the merger rate (Stewart 2009; Hopkins et al. 2010). This bias against gas-rich satellites at high- $z$ must be accounted for, when inferring the contribution of galaxy mergers to the cosmic star formation rate density.

In light of these considerations, we restrict ourselves to drawing conclusions on the stellar mass accretion rate and size evolution of passive galaxies. Our measurements indicate that on average, a massive galaxy doubles its stellar mass from $z \sim 3$ to 0.3 by accreting stars, mainly through major mergers. The observed merger rate is sufficient to explain the number density evolution of the most massive galaxies $\left(M_{\star} \geqslant 10^{11.1} M_{\odot}\right)$. Meanwhile, the observed major and minor merger rates can only explain half of the size evolution observed for massive passive galaxies during $z=0-3$ (Fig. ; Newman et al. 2012; van der Wel et al. 2014). This implies that additional mechanisms (e.g., progenitor bias, Carollo et al. 2013) are required to explain the rapid size growth of the passive galaxy population.

\section{References}

Bluck, A. F. L., et al. 2009, MNRAS, 394, L51

Carollo, C. M., et al. 2013, ApJ, 773, 112

Grogin, N. A., et al. 2011, ApJS, 197, 35

Hopkins, P., et al. 2010, ApJ, 724, 915

Ilbert, O., et al. 2013, A\& $A, 556, \mathrm{~A} 55$

Kartaltepe, J. S., et al. 2010, ApJ, 721, 98 
Koekemoer, A. M., et al. 2011, ApJS, 197, 36

Lotz, J. M., Jonsson, P., Cox, T. J., \& Primack, J. R. 2010, MNRAS, 404, 575

Man, A. W. S., Toft, S., Zirm, A. W., Wuyts, S., \& van der Wel, A. 2012, ApJ, 744, 85

Man, A. W. S., et al. 2014, ApJ, submitted; arXiv:1410.3479

McCracken, H. J., et al. 2012, A\&A, 544, A156

Naab, T., Johansson, P. H., \& Ostriker, J. P. 2009, ApJ, 699, L178

Newman, A. B., Ellis, R. S., Bundy, K., \& Treu, T. 2012, ApJ, 746, 162

Stewart, K. R. 2009, 'Invisible Major Mergers: Why the Definition of a Galaxy 'Merger Ratio'

Matters," in Galaxy Evolution: Emerging Insights and Future Challenges, eds. S. Jogee,

I. Marinova, L. Hao, \& G. A. Blanc (ASP: San Francisco) p. 243

Treister, E., Schawinski, K., Urry, C. M., \& Simmons, B. D. 2012, ApJ, 758, L39

Williams, R. J., Quadri, R. F., \& Franx, M. 2011, ApJ, 738, L25

van der Wel, A., et al. 2014, ApJ, 788, 28 